\title{
Mapeo del riesgo: Prioridades para prevenir el establecimiento de tamariscos invasores
}

\author{
Evangelina Natale ${ }^{1, \bigotimes}$; Herminda E. Reinoso; ${ }^{1}$ Verónica AndreO ${ }^{1,2}$ \& Sergio M. \\ $\mathrm{ZALBA}^{3}$
}

${ }^{1}$ Departamento de Ciencias Naturales. Universidad Nacional de Río Cuarto. Córdoba, Argentina. ${ }^{2}$ Department of Earth Observation Science. ITC. Faculty of Geo-Information Science and Earth Observation. University of Twente. Enschede, The Netherlands. ${ }^{3}$ Grupo de Estudios en Conservación y Manejo. Departamento de Biología, Bioquímica y Farmacia, Universidad Nacional del Sur.

\begin{abstract}
Resumen. La detección precoz y la intervención rápida se encuentran entre las acciones más apropiadas para lograr resultados exitosos en los planes de manejo de especies exóticas invasoras. En este marco, la identificación de áreas donde las probabilidades de establecimiento e invasión de una especie sean altas puede disminuir los costos y los esfuerzos logísticos y económicos del manejo. La evaluación de riesgo ha adquirido gran aceptación como metodología basada sobre información de la especie exótica invasora y del ambiente receptor para estimar la probabilidad y la magnitud de la amenaza. El objetivo de este trabajo fue desarrollar un índice de riesgo de invasión capaz de modelar, en un entorno de sistema de información geográfica (SIG) y combinando información de la distribución actual y potencial de una especie, el análisis de rutas de dispersión y el impacto potencial sobre valores ambientales y socioeconómicos. Se utilizó como estudio de caso el proceso de invasión por el género Tamarix, cuyas especies se distribuyen desde el norte de la Patagonia argentina hasta el noroeste del país. Como resultado se obtuvo un índice de riesgo con potencial para ser expresado cartográficamente en un SIG y apropiado para aplicarse a distintas especies invasoras, regiones y escalas.
\end{abstract}

[Palabras clave: análisis de riesgo, Tamarix, tamariscos, Argentina, conservación, biodiversidad]

\begin{abstract}
Aвstract. Mapping risk: Priorities to prevent the establishment of invasive tamariscs. Early detection and rapid intervention are among the preferred actions to achieve successful results in the management plans for invasive alien species. The identification of areas where probabilities of establishment and invasion by an alien species are high can reduce logistic and economic costs. Risk assessment based on information about the alien species and the invaded environment has become a widely-accepted tool to estimate the likelihood and magnitude of the threat. The objective of this work was to develop an invasion risk index that can be modeled within a geographic information system (GIS) environment, combining information on the current and potential distribution of the alien species, the analysis of dispersion routes and the potential impact on environmental and socioeconomic values. The genus Tamarix was used as a case study. This genus is widely distributed in Argentina, from northern Patagonia to the northwest of the country. A risk index was obtained, with potential to be expressed cartographically in a GIS. The relevance of the developed index is that it might be extended and applied to different invasive species, regions and working scales.
\end{abstract}

[Keywords: risk analysis, Tamarix, saltcedar, Argentina, conservation, biodiversity]

\section{INTRODUCCIÓN}

Las posibilidades de controlar de manera eficiente un proceso de invasión decrecen con el paso del tiempo desde que una especie es introducida en una nueva región, en función del aumento de su abundancia y del área cubierta. Por eso, la detección precoz y la acción rápida están entre las opciones de manejo preferidas. Sin embargo, detectar organismos invasores en baja densidad en territorios extensos es un desafío en sí mismo y entraña costos muy elevados, a menudo incompatibles con los presupuestos de las agencias de manejo. Identificar áreas donde las probabilidades de establecimiento e invasión de la especie sean altas puede disminuir los

Editor asociado: Martín Nuñez costos y el esfuerzo asociado a una estrategia de control temprano (Zalba et al. 2000; Andreu and Vilá 2010; Kaplan et al. 2014).

Existen herramientas metodológicas que permiten predecir la probabilidad de establecimiento de una especie en un área, así como su distribución potencial en una región. Sin embargo, desde el punto de vista de los objetivos de conservación importa identificar aquellas áreas donde la especie no sólo pueda prosperar, sino también donde los efectos sobre el ambiente puedan resultar significativos (Andreu and Vilá 2010). Desde una perspectiva práctica, lo que se debería evaluar es el riesgo asociado a una invasión. Para ello es necesario combinar a) información 
sobre la probabilidad de que la especie consiga establecerse y prosperar en una región, con b) las consecuencias socioeconómicas y ambientales previsibles a partir de su presencia allí (Mack et al. 2002).

La evaluación de riesgo está aceptada como una metodología basada en información sobre la especie exótica invasora y el ambiente receptor, y permite estimar la probabilidad y la magnitud de la amenaza (Stohlgren and Schnase 2006; Neville and Bass 2008; Singh et al. 2014). Como resultado de este análisis se pueden detectar sitios donde la probabilidad de establecimiento de una especie invasora es moderada, pero con consecuencias que pueden tener un alto riesgo socioambiental, y sitios con alta probabilidad de establecimiento, pero con consecuencias menores, que suelen traducirse en un riesgo bajo (Mack et al. 2002). Así, estos tipos de estudios se convierten en una herramienta que les permite a los planificadores identificar sitios donde es prioritario monitorear nuevos focos de invasión e intervenir proactivamente (Foxcroft et al. 2006).

Por medio de algoritmos matemáticos o estadísticos, los sistemas de información geográfica (SIG) pueden combinar datos georeferenciados de localización de una especie invasora con variables ambientales locales para crear un modelo de requerimientos en esas dimensiones ambientales y proyectarlo sobre un espacio geográfico determinado (Porcasi et al. 2005; Ward 2007). Esto permite plasmar los resultados de las evaluaciones de riesgo en diferentes escalas geográficas, siempre según el nivel de resolución de la información que alimente al modelo. De esta manera se pude abordar la problemática tanto en grandes espacios geográficos (e.g., un país) como en escalas locales (e.g., un área protegida, que es la unidad de manejo más utilizada en las estrategias de conservación de la biodiversidad).

El objetivo de este trabajo fue diseñar un índice de riesgo de invasión en una plataforma de entorno SIG, combinando la información sobre la distribución actual de una especie invasora con modelos de distribución potencial, análisis de rutas de dispersión e impacto potencial sobre valores ambientales y socioeconómicos. El diseño se elaboró utilizando como caso de estudio al género Tamarix, con el que los autores están familiarizados y cuyas especies invasoras se distribuyen extensamente en la Argentina.

\section{La invasión de tamariscos en la Argentina}

El género Tamarix incluye un conjunto de especies nativas de Asia, del norte de África y del sudeste de Europa, entre las que se cuentan algunas de las invasoras de mayor impacto en ambientes ribereños de Estados Unidos, México y Australia (De Loach et al. 2000; Hart et al. 2005; Australia Weeds Committee 2007). En Sudamérica, un conjunto de especies de este género se estableció en Argentina, Brasil y Uruguay (Natale et al. 2008; Zalba comunicación personal). En la Argentina se citan cuatro especies de tamarisco (Tamarix gallica, $T$. chinensis, $T$. ramosissima y $T$. parviflora), que crecen de manera espontánea en ambientes naturales y seminaturales del centro-oeste y del sureste del país (Natale et al. 2008). Su dispersión siguió un patrón similar a los reportados para Estados Unidos, Australia y México, ya que colonizaron principalmente ambientes ribereños de zonas áridas y semiáridas, continentales y costeras (Natale et al. 2010). Sin embargo, y a pesar de que la mayor parte del territorio argentino corresponde a regiones áridas y semiáridas, la magnitud del problema de invasión por tamariscos no parece ser, a la fecha, tan grave como la reportada en el resto de los países invadidos. Esto podría deberse a que el proceso de invasión se encuentra en una fase inicial, eventualmente retardado por un menor número de focos iniciales de invasión y/o por una menor densidad de rutas de dispersión (Natale et al. 2012). En consecuencia, se trata de una situación propicia para desarrollar estrategias de prevención que reduzcan las chances de expansión del género (Natale et al. 2013).

\section{Impactos ambientales y socioeconómicos de las invasiones de tamarisco}

La invasión por tamariscos ha sido vinculada a varios efectos negativos que pueden alterar profundamente tanto la composición de especies como los procesos ecosistémicos (De Loach et al. 2000). Sin embargo, las pruebas de que Tamarix constituye la principal o la única causa de estos efectos es ambigua (Shafroth and Briggs 2008). Entre los impactos que se le atribuyen a éste género se pueden citar: 1) el aumento de la frecuencia y la gravedad de las inundaciones, ya que el estrechamiento de los ríos producido por los parches densos de tamariscos disminuye la capacidad de contención de agua (Cleverly et al. 1997; Smith et al. 1998; De Loach et al. 2000; Zavaleta 2000); 2) la reducción de la disponibilidad de agua 
(estudios en distintos ríos mostraron niveles de evapotranspiración entre 0.75 y $1.45 \mathrm{~m} /$ año, asociados a la presencia de tamariscos [Cleverly et al. 2002; Owens and Moore 2007; Nagler et al. 2008]); 3) el aumento de la salinidad de los suelos (los antecedentes muestran una correlación fuerte entre el avance de los tamariscos y la desertificación y la salinización de las cuencas; esto resulta en la extinción local de la vegetación nativa y, eventualmente, en una dominancia completa de las planicies de inundación por bosques monoespecíficos de la invasora [Lawrence and Stanley 1997; Cleverly et al. 1997; Smith et al. 1998]); 4) los cambios en la dinámica del fuego (se ha observado que los fuegos naturales son raros en las comunidades de plantas riparias, pero en áreas invadidas por tamariscos se vuelven más frecuentes y más destructivos [Lovich 1996; De Loach et al. 2000]); 5) el reemplazo y empobrecimiento de la vida silvestre (los parches monoespecíficos brindan poca diversidad estructural y microclimática, lo que redunda en una disminución de la diversidad de aves [van Riper et al. 2008; Stromberg et al. 2009]); 7) la reducción del valor recreacional (ya que los tamariscos condicionan el uso recreativo de las costas de los ríos, lagos y lagunas en parques, refugios de vida silvestre y otras áreas usadas para acampar, cazar, pescar, navegar y para safaris fotográficos [De Loach et al. 2000], y 8) la caída del valor productivo de la tierra (el avance del tamarisco se asocia al empobrecimiento del forraje, a la disminución del agua de riego y al incremento de la salinidad del suelo y de la frecuencia de los incendios). Sumado a esto, las especies del género resultan de baja calidad en cuanto a la provisión de sombra durante el verano (De Loach et al. 2000). Además, comprometen los servicios de polinización (Zavaleta 2000) dado que reducen la diversidad y la abundancia de insectos.

\section{Materiales y Métodos}

En primer lugar, para construir el índice de riesgo se realizó una búsqueda de información cartográfica sobre las diferentes variables que se iban a utilizar.

\section{Valores socioeconómicos y de conservación sensibles a la presencia de Tamarix spp.}

Dada la capacidad de la especie de colonizar ambientes ribereños y de alterar la estructura de los hábitats asociados, se identificaron ambientes vulnerables a partir del mapa de distribución de humedales de la Base Ecológica para la Clasificación de Humedales en Argentina (Malvárez and Bó 2004). También se consideraron otros valores naturales sensibles al impacto de la invasión por tamariscos (e.g., cursos de agua), áreas clave para conservar la biodiversidad a nivel nacional (e.g., áreas de importancia para la conservación de las aves [AICAs] [Di Giacomo 2005]) y áreas valiosas de pastizal (AVPs) (Bilenca and Miñarro 2004). A esta información se le sumo la capa del Sistema Federal de Áreas Protegidas de Argentina y la de ecorregiones de Argentina, priorizadas de acuerdo a su grado de amenaza (Burkart et al. 1996; Bertonatti and Corcuera 2000; Brown et al. 2005).

Para detectar los valores socioeconómicos que se podrían ver afectados por la invasión de tamariscos se utilizó la variable tipo de suelo, obtenida del Atlas de Suelos de la República Argentina (INTA 1995) e información sobre la capacidad de uso de la tierra (Agencia Córdoba Ambiente 2003). Se consideraron las áreas que presentan buena aptitud para uso agrícolo-ganadero, las que, en caso de ser invadidas por tamariscos, disminuyen su valor productivo por el incremento de los costos de producción que implica el control de la invasión. Finalmente, también se consideraron dentro de esta categoría las obras de infraestructura hídrica que podrían verse afectadas por la presencia de Tamarix.

\section{Datos de distribución de tamariscos en la Argentina}

Para determinar las áreas potencialmente invasibles por el género Tamarix se utilizaron los mapas de distribución actual y potencial de tamarisco en la Argentina realizados por Natale et al. (2008 y 2013, respectivamente). La distribución potencial del género en el país se tomó de un modelo de ajuste climático el obtenido con el algoritmo DOMAIN con 12 variables climáticas (Natale et al. 2013), por considerarlo el modelo con mayor poder de predicción. Dicho modelo utilizó como puntos de entrenamiento los sitios donde la especie era considerada invasora y/o establecida (es decir que el núcleo poblacional presentaba extensiones grandes y una estructura etaria heterogénea [adultos, juveniles y plántulas]). Se trabajó con una resolución espacial de 30 arc-segundos $(\sim 1 \times 1 \mathrm{~km})$. Esto significa que el máximo acercamiento fue en un área aproximada de 10000 ha, lo que brinda un detalle elevado, ya que la mayoría de las unidades de manejo en la Argentina son 
áreas protegidas que poseen una superficie equivalente o mayor.

\section{Rutas de dispersión y preferencias de hábitat de Tamarix spp.}

Los vectores y las rutas de dispersión se obtuvieron de Natale et al. (2012) dado que consideran a los cursos de agua y a las vías de comunicación (i.e., caminos y ferrocarriles) como las principales vías de dispersión del género. En cuanto a la preferencia de hábitat, se tuvo en cuenta la importancia de las inundaciones para el reclutamiento de estas especies (Natale et al. 2016) y otros antecedentes sobre variaciones en su comportamiento invasor en otras regiones de mundo (Horton et al. 2001; Glenn and Nagler 2005), por lo que se incluyeron en el análisis las variables riesgo de inundación, drenaje y tipo y textura de suelo, obtenidas del Atlas de Suelos de Argentina (INTA 1995).

\section{Construcción del índice de riesgo}

Para la obtención del índice de riesgo, a la condición de cada variable seleccionada se le asignó un valor numérico. Así, para los valores de conservación, a la condición más

Tabla 1. Categorías de las variables utilizadas en el desarrollo del índice de riesgo de invasión de tamariscos (Tamarix spp.) en la Argentina (Stromberg et al. 2009; Owens and Moore 2007; Cleverly et al. 2002; Natale 2010; Nagler et al. 2008; Natale et al. 2010; Sobral and Nakama 1990; Zavaleta 2000; Natale et al. 2012; Van Ripper et al. 2008).

Table 1. Categories of the variables used in the development of the index of invasion risk for saltcedar (Tamarix spp.) in Argentina (Stromberg et al. 2009; Owens and Moore 2007; Cleverly et al. 2002; Natale 2010; Nagler et al. 2008; Natale et al. 2010; Sobral and Nakama 1990; Zavaleta 2000; Natale et al. 2012; Van Ripper et al. 2008).

\begin{tabular}{|c|c|c|c|}
\hline Variables & & Categorías & Descripción \\
\hline \multirow[t]{4}{*}{$\begin{array}{l}\text { Variables } \\
\text { de hábitat }\end{array}$} & Altitud & $\begin{array}{l}1=<2000 \text { m s. n. m. } \\
0=>2000 \text { m s. n. m. }\end{array}$ & $\begin{array}{l}\text { De acuerdo al comportamiento del género en la Argentina, } \\
\text { donde no se registran casos de invasión por encima de los } 2000 \\
\text { m s. n. m. pese a la presencia de la especie. }\end{array}$ \\
\hline & Tipo de Suelo & $3=$ Molisol y Entisol & De acuerdo al comportamiento del género registrado en la \\
\hline & & $\begin{array}{l}2=\text { Roca }, \text { Aridisol y Alfisol } \\
1=\text { Vertisol, Haplustol, etc. } \\
0=\text { sin información }\end{array}$ & Argentina y en otras regiones del mundo. \\
\hline & $\begin{array}{l}\text { Disponibilidad } \\
\text { Hídrica }\end{array}$ & & $\begin{array}{l}\text { Se asume que las zonas con mayor cobertura de humedales son } \\
\text { más susceptibles debido a la abundancia de sitios apropiados } \\
\text { para la germinación y el establecimiento de Tamarix spp. }\end{array}$ \\
\hline \multirow[t]{5}{*}{$\begin{array}{l}\text { Valores } \\
\text { naturales }\end{array}$} & $\begin{array}{l}\text { Sistema Federal } \\
\text { de Áreas } \\
\text { Protegidas (AP) }\end{array}$ & $\begin{array}{l}1=\text { área protegida } \\
0=\text { ausencia }\end{array}$ & Áreas de alto valor para la biodiversidad bajo protección legal. \\
\hline & AICA & $\begin{array}{l}1=\text { AICA } \\
0=\text { ausencia }\end{array}$ & Áreas de alto valor de conservación para las aves. \\
\hline & AVPs & $\begin{array}{l}1=\text { AVP } \\
0=\text { ausencia }\end{array}$ & Pastizales naturales con alto valor de conservación. \\
\hline & Humedales & $\begin{array}{l}3=\mathrm{AIH} \\
2=\mathrm{APH} \\
1=\mathrm{NH} \\
0=\text { ausencia }\end{array}$ & $\begin{array}{l}\text { Humedales de alto valor de conservación reconocido a nivel } \\
\text { internacional por la convención Ramsar (Malvárez and Bó 2004). }\end{array}$ \\
\hline & Ecorregiones & $\begin{array}{l}3=\mathrm{Es}, \mathrm{Lp}, \mathrm{ML} \text { y } \mathrm{M} \\
2=\mathrm{MS} \text { y }, \mathrm{AA} \\
1=\mathrm{CS}, \mathrm{Pu}, \mathrm{EP} \\
0=\text { resto de las ecorregiones }\end{array}$ & $\begin{array}{l}\text { Nivel de organización biológica más apropiado para conservar } \\
\text { la variabilidad de las especies, los ecosistemas y sus funciones } \\
\text { (escala de paisaje) (Brown et al. 2006). La valoración respondió a } \\
\text { la amenaza actual en que se encuentra cada una de las regiones } \\
\text { según Bertonatti and Corcuera (2000) y cómo son afectadas por } \\
\text { la presencia de Tamarix. }\end{array}$ \\
\hline \multirow[t]{4}{*}{$\begin{array}{l}\text { Valor } \\
\text { productivo }\end{array}$} & IPC & $\begin{array}{l}5=\text { clase I } \\
4=\text { clases II y III } \\
3=\text { clases IV y V } \\
2=\text { clases VI y VII } \\
1=\text { clases VIII y IX } \\
0=\text { sin información }\end{array}$ & $\begin{array}{l}\text { Las clases por capacidad de uso hacen referencia al grado de } \\
\text { limitación agrícola y pastoril. Los riesgos de daño al suelo o } \\
\text { limitaciones en su uso se hacen progresivamente mayores desde } \\
\text { la clase I a la IX. }\end{array}$ \\
\hline & Infraestructura & $3=$ canal de riego & Por antecedentes de impacto en otras regiones del mundo y \\
\hline & $\begin{array}{l}\text { cursos de agua } \\
\text { (IHCURA) }\end{array}$ & $\begin{array}{l}1=\text { cursos de agua sin } \\
\text { infraestructura } \\
0=\text { sin cursos de agua }\end{array}$ & rque son luente uer recurso agua. \\
\hline & $\begin{array}{l}\text { Infraestructura } \\
\text { hídrica en } \\
\text { cuerpos de agua } \\
\text { (IHCUE) }\end{array}$ & $\begin{array}{l}4=\text { embalses/diques } \\
3=\text { lagos/lagunas } \\
2=\text { aguadas/manantial } \\
1=\text { bañados } \\
0=\text { sin cuerpo de agua }\end{array}$ & $\begin{array}{l}\text { Por antecedentes de impacto en otras regiones del mundo y } \\
\text { porque son fuente del recurso agua. }\end{array}$ \\
\hline \multirow[t]{2}{*}{$\begin{array}{l}\text { Vectores } \\
\text { y rutas de } \\
\text { dispersión }\end{array}$} & $\begin{array}{l}\text { Vías de } \\
\text { comunicación }\end{array}$ & $\begin{array}{l}1=\text { presencia } \\
0=\text { ausencia }\end{array}$ & $\begin{array}{l}\text { De acuerdo con lo analizado, los caminos y ferrocarriles } \\
\text { actuarían como vías de dispersión complementarias a los cursos } \\
\text { de agua. }\end{array}$ \\
\hline & Cursos de agua & $\begin{array}{l}2=\text { permanentes } \\
1=\text { transitorios } \\
0=\text { ausencia }\end{array}$ & Según antecedentes, es la principal vía de dispersión del género. \\
\hline
\end{tabular}


vulnerable en cada caso se le asignó el valor más alto y a la de menor riesgo, el valor nulo. En el caso de los valores socioeconómicos, a los suelos con mayor aptitud de uso, así como a las obras de mayor envergadura se les asignó el valor máximo. Las rutas de dispersión y las distribuciones actuales y potenciales se transformaron en variables binarias. Para el caso de las variables de hábitat, los valores altos fueron asignados a las condiciones que son favorables para la presencia del género (Tabla 1). Una vez traducidas en valores numéricos, las variables fueron relacionadas a través de algoritmos matemáticos para construir un índice global que permita identificar situaciones en las que la presencia de Tamarix implique riesgos ambientales y socioeconómicos altos, medios o bajos.

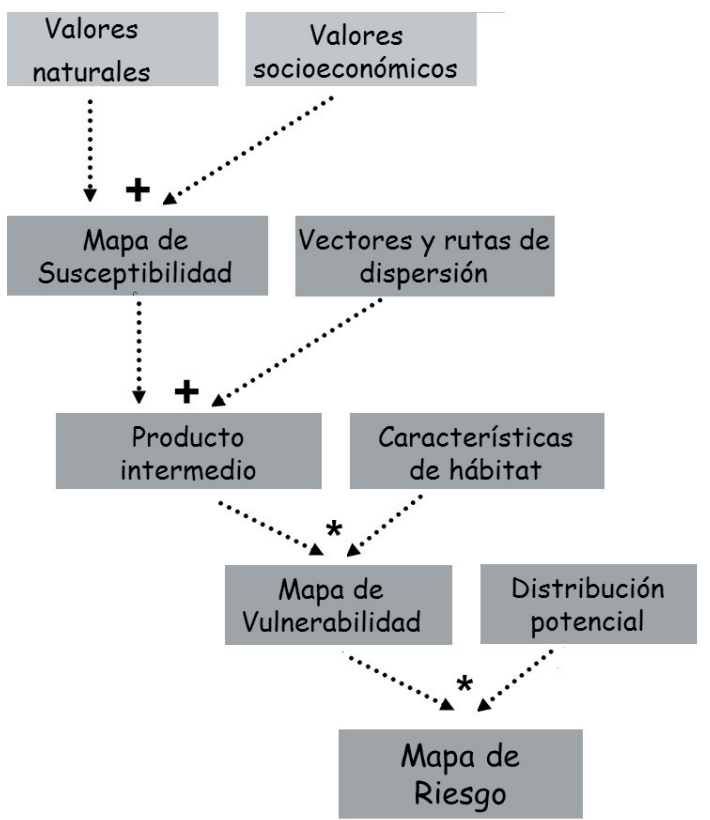

Figura 1.Combinación de las variables seleccionadas para la construcción de un índice de riesgo de invasión por tamariscos en la Argentina.

Figure 1. Combination of the variables used for the construction of an index of invasion risk by saltcedars in Argentina.
Por último, todas las variables se tradujeron en capas de información dentro de una plataforma de sistema de información geográfica. Las capas de vectores se interpolaron a formato raster (Chen et al. 2007) para estandarizar la información y se empleó la extensión de análisis espacial del software Arcgis 9.10 (ESRI, Redlands, CA, USA) para relacionar las capas de información a través de los algoritmos matemáticos seleccionados y obtener el mapa de riesgo final.

\section{Resultados}

Índice de riesgo de invasión para Tamarix spp. en la Argentina

A partir de la combinación de los valores naturales y socioeconómicos se obtuvo un índice de sensibilidad (Figura 1), entendido como el grado de fragilidad del sistema frente al establecimiento y la invasión de tamariscos. Así, las variables consideradas para estimar el valor natural y socioeconómico se combinaron a través de la media aritmética, de tal manera que un valor nulo (0) de cualquiera de las variables se podría ver compensado por el valor de otra (ecuaciones a y b). Para obtener el índice de sensibilidad, ambos valores se relacionaron a través de una sumatoria (ecuación c), ya que mientras más valores posea el área, mayor será su fragilidad frente al establecimiento del tamarisco. De esta forma, el índice de sensibilidad representaría tres situaciones diferentes: 1) áreas de sensibilidad alta donde coincidan elevados valores naturales y socioeconómicos; 2) áreas de sensibilidad intermedia donde se podrían encuadrar áreas con muy alto valor de alguno de los dos aspectos o áreas con valores intermedios de ambos aspectos; y 3) áreas menos sensibles, con bajos valores para los dos aspectos considerados. De acuerdo a los valores expresados en la Tabla 1, este índice oscilaría entre 0 (valor nulo) y un máximo de 6.25 .

Valores naturales $(\mathrm{VN})=(\underline{\mathrm{AP}+\mathrm{AICA}+\mathrm{AVP}+\mathrm{Ecorregió}+\text { Humedales })}$

Ecuación a

4

Valores socioeconómicos $(\mathrm{VSE})=\underline{(\mathrm{IPC}+\mathrm{IHCURA}+\mathrm{IHCUEA})}$

Ecuación b

3

Índice de sensibilidad (IS) $=\mathrm{VN}+\mathrm{VSE}$

Ecuación c

Variables de hábitat $(\mathrm{VH})=\underline{\text { (altitud+tipos de suelo) }}$

Ecuación d

Vectores y rutas de dispersión (VD) = (cursos de agua+vías de comunicación)

Ecuación e

Índice de vulnerabilidad $(\mathrm{IV})=(\mathrm{IS}+\mathrm{VD})^{*} \mathrm{VH}$

Ecuación $\mathrm{f}$ 
Como segunda instancia se generó un índice de vulnerabilidad, definido como la probabilidad de que el sistema se vea afectado por la invasión, considerando 1) su sensibilidad al impacto, calculada con el índice de sensibilidad antes obtenido (IS), 2) su accesibilidad para la especie, considerando la presencia de vectores y rutas de dispersión, y 3) la aptitud del ambiente para ser colonizado (Figura 1). Dado que el mapa de distribución potencial usado en este estudio (Natale et al. 2013) sólo contempla variables climáticas, fue necesario incluir otras variables de hábitat (e.g., altitud y tipo de suelo), a las que se consideró fundamentales para el establecimiento y la generación del proceso de invasión de tamarisco.

Los vectores y rutas de dispersión (VD) (Natale et al. 2012) y las variables de hábitat se relacionaron entre sí a través de la media aritmética para reflejar una relación compensatoria (ecuaciones d y e). Así, valores bajos de una de las variables pueden ser compensados por valores altos de la otra $y$, por lo tanto, ninguna de las variables seleccionadas resulta limitante ni para la dispersión ni para el establecimiento del tamarisco en un sitio determinado. Luego, el índice de sensibilidad (IS) fue relacionado con las rutas de dispersión (VD) a través de una sumatoria que refleja las mayores posibilidades de llegada del género en los casos de presencia de vías de dispersión (exposición), sin descartar la posibilidad de que los tamariscos puedan dispersarse de forma natural a través del viento y el agua o ser introducidos deliberadamente por el ser humano. El resultado de esta sumatoria se relacionó con variables de hábitat $(\mathrm{VH})$ a través de un producto, de forma tal que los sitos con baja o nula aptitud para la presencia del género reciben valores bajos o nulos de vulnerabilidad (ecuación $\mathrm{f}$ ). Es decir que si el sitio donde logró dispersarse el tamarisco no presenta las condiciones mínimas necesarias que permitan su establecimiento, no se encontraría bajo amenaza.

Finalmente, para la obtención del índice de riesgo se combinó el índice de vulnerabilidad (IV) con el mapa de distribución potencial de tamarisco (MDP), entendido como la probabilidad de presencia del género. El algoritmo utilizado en este caso fue el producto entre el índice de vulnerabilidad (IV) y el mapa de distribución potencial (MPD), con la finalidad de que las celdas que presentaran valor 0 para alguno de los componentes tuvieran también valor nulo en el índice de riesgo final. Es decir que sólo las áreas vulnerables con probabilidades de que el género se desarrolle son consideradas en el análisis del riesgo de invasión (ecuación g).

\section{Índice de riesgo (IR): IV*MDP Ecuación g}

Como resultado final se obtuvo un índice que osciló entre 0 (riesgo nulo) y 32 (riesgo muy alto).

\section{Mapa de riesgo de invasión para Tamarix spp. en la Argentina}

En las Figuras 2 y 3 se muestran los mapas intermedios obtenidos y el mapa final de riesgo. Para facilitar la lectura del mapa final, el rango de valores de índice de riesgo se fraccionó en cuatro intervalos: 0-0.9 (nulo), 1-5 (bajo), 5.1-13 (medio) y 13.1-32 (alto).

El mapa de riesgo obtenido para Tamarix spp. en la Argentina (Figura 3) logró discriminar zonas de alto, medio y bajo riesgo dentro del área de probabilidad de presencia del género (Natale et al. 2012). Por ejemplo, se puede observar como en el este de Buenos Aires y en el norte de La Pampa encontramos zonas de alto riesgo de invasión, rodeadas por sectores de riesgo medio, mientras que las zonas de riesgo bajo quedaron circunscriptas principalmente al oeste de San Juan y la Rioja.

\section{Comprobación del índice de riesgo con datos de campo}

En la Figura 4a se muestra que el sur de la provincia de Córdoba es un área de distribución potencial de Tamarix donde, a su vez, existen registros actuales de presencia de poblaciones del género en distintas categorías de invasión, de acuerdo a lo publicado por Natale et al. (2012). Los resultados arrojados por el índice de riesgo en dicha zona (Figura $4 b)$ permiten discriminar áreas con riesgos altos, medios y bajos. A modo de validación, y analizando los pasos intermedios hasta llegar al mapa de riesgo, es posible observar que las zonas de bajo riesgo se corresponden con sitios de nulo valor productivo (Figura 4c), pero valores medios de conservación (Figura 4d). Esto se debe a que allí se encuentran los bañados del río Saladillo, áreas inundables y salinas poco productivas, pero que conforman un complejo de humedales clave para la preservación de aves acuáticas (López et al. 2013). Las zonas identificadas como de alto riesgo se justifican con la presencia de sitos con valores productivos medios a altos y con valores de conservación medios. El sureste de 

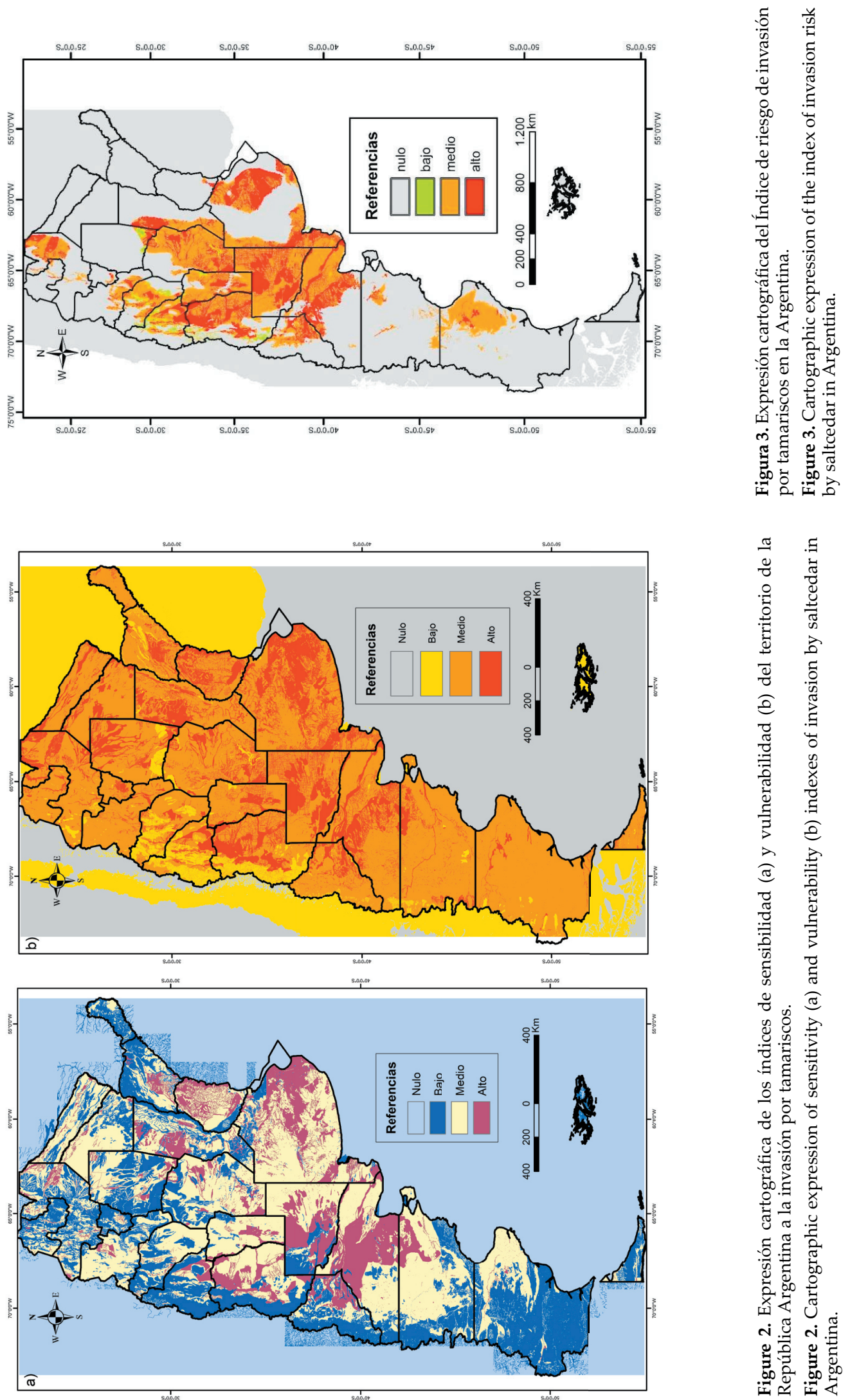

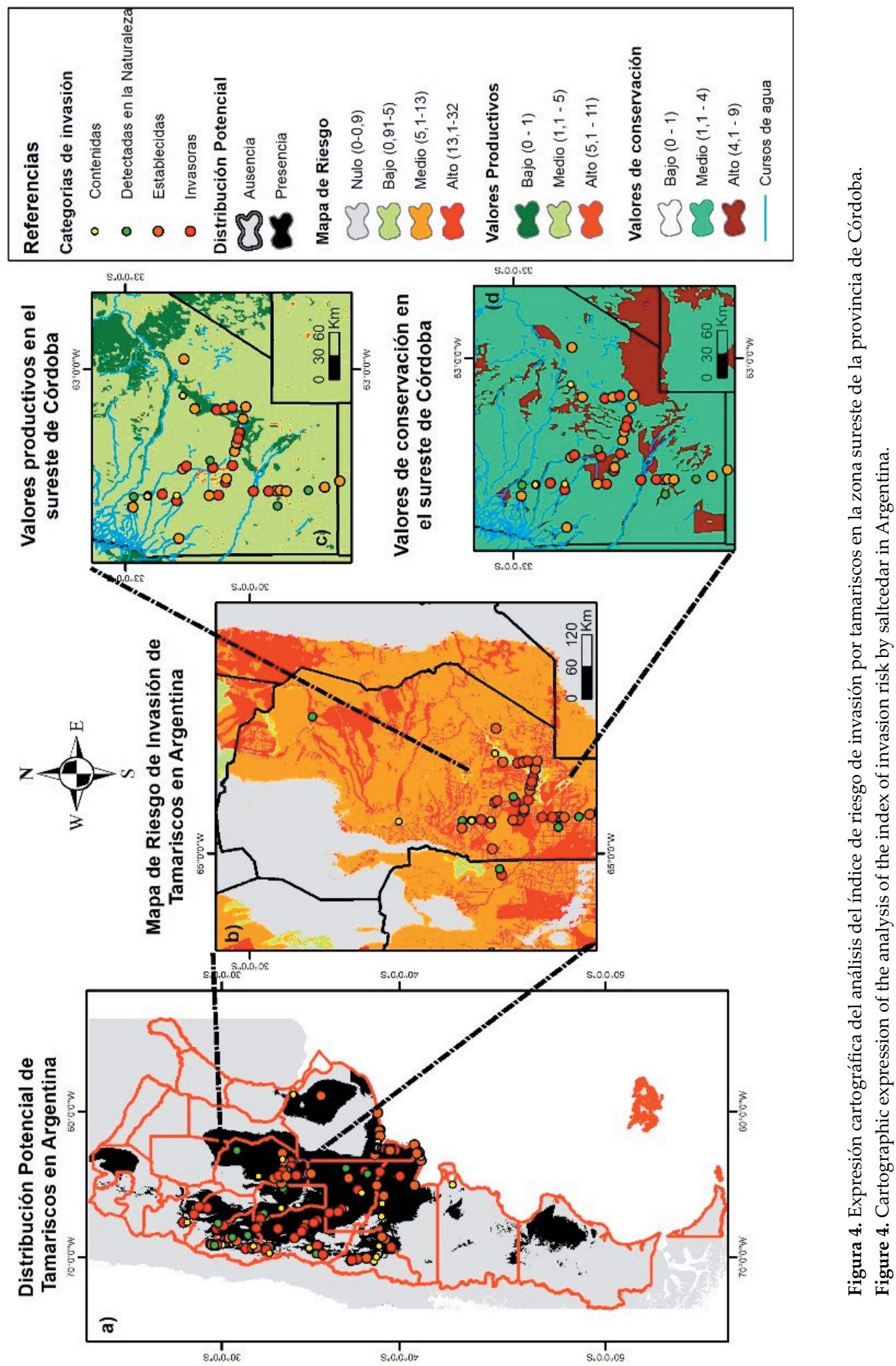
la provincia de Córdoba es una de las zonas agropecuarias más productivas (López et al. 2013). Esta situación disminuye el valor de conservación, ya que el cambio de uso del suelo llevó a la pérdida casi total de los ambientes naturales, relegados a sitios marginales de baja productividad (Oggero et al. 2014). Lo único que eleva el valor de conservación de esta zona es que pertenecen a la Región del Espinal, considerada a nivel nacional como una de las regiones más amenazadas por la actividad antrópica y, por lo tanto, de alta prioridad de conservación (Bertonatti and Corcuera 2000; Brown et al. 2006). Cabe destacar que la superposición de los sitios relevados con poblaciones de tamariscos en distintos grados de invasión permite, a su vez, definir áreas prioritarias dentro de las diferentes zonas de riesgo. Este análisis de un área particular ampliamente estudiada y conocida da cuenta de la validez y confiabilidad del índice que se presenta en este trabajo. No obstante, la confiabilidad general de estos resultados está sujeta a la precisión y la escala en la que se encuentra la información susceptible de ser mapeada. En este caso en particular, la mayor parte de la información está en una escala de 1:250.000. En el caso particular del mapa de distribución potencial, que constituye uno de los inputs del índice de riesgo, de acuerdo a lo reportado por Natale et al. (2013), presentó un AUC de 0.86, un error de omisión de 1.30\% y una sensibilidad de $98.3 \%$.

\section{Discusión y Conclusiones}

La mayoría de las evaluaciones de riesgo, como la presentada por Kapplan et al. (2014), sólo trabaja sobre un marco para mejorar la detección de poblaciones de plantas naturalizadas, a fin de delimitar con precisión su presencia en un sitio determinado. El sistema de análisis de riesgo desarrollado en este trabajo resulta novedoso y atiende a las demandas sugeridas por Kumschick and Richardson (2013) dado que combina información referida a la expansión de las especies exóticas invasoras con información sobre el impacto ambiental (valores de conservación) y el impacto socioeconómico (valor productivo) que puede producir en el área evaluada. También se diferencia de otras iniciativas en las que se pone énfasis sólo en los riesgos sobre la agricultura y la producción forestal (Ou et al. 2008). La conservación de la biodiversidad requiere que una priorización efectiva, aunque sólo sea porque los fondos son limitados y se deban asignar cuidadosamente [Marchese 2015]). Por eso, la principal aplicación de este índice es concentrar esfuerzos de detección precoz y erradicación en áreas que combinan chances altas de colonización con un elevado impacto actual o potencial, a bajos costos económicos y/o logísticos. Estos protocolos de evaluación de prioridades de manejo les brindan a los agentes de conservación productos de gestión informativo-cartográficos para el manejo del riesgo (Andersen et al. 2004). También incluyen la toma de decisiones relativas a la introducción de especies, sus vectores y rutas de dispersión (Foxcroft et al. 2006). Desde este punto de vista, y en coincidencia con Kapplan et al. (2014) y Kumschick and Richardson (2013) sobre la necesidad de definir protocolos de búsqueda sistemática que permitan una delimitación rápida de todos los sitios con invasiones comprobadas y sitios que merezcan generar protocolos de vigilancia para detectar cualquier nueva población, este índice permitió categorizar al territorio nacional en diferentes situaciones de riesgo. Por un lado, se identificaron sitios con registros confirmados del género; éstos deberán estar sujetos a acciones de erradicación local, control y/o contención, con acciones más urgentes en las zonas de alto riesgo. De todas maneras, no se debe minimizar la vigilancia en las situaciones de bajo riesgo con presencia confirmada de poblaciones invasoras que podrían ser fuentes de propágulos hacia zonas de alto riesgo aún no colonizadas. Otra situación identificada corresponde a las zonas de alto riesgo sin presencia confirmada del invasor, donde deben plantearse estrategias de detección temprana y control precoz.

En cuanto a los aspectos metodológicos presentados en este trabajo, resulta novedoso el uso de algoritmos matemáticos siguiendo la lógica de los índices que se utilizan para evaluar calidad de hábitat (Chen et al. 2009; Burgman et al. 2001). Esto genera una valoración cuantitativa del territorio y lo divide en un conjunto de pequeñas subunidades, tal que cada unidad puede ser calificada por la posibilidad de que la especie invasora se establezca allí y cause un impacto mensurable. Esto lo diferencia de otras evaluaciones de riesgo que se basan en árboles de decisión (Kapplan et al. 2014), modelos climáticos de distribución de especies (Pertierra et al. 2016) o modelos semicuantitativos y/o sistemas de puntuación (Davidson et al. 2017; Drolet et al. 2016). Otra diferencia importante de este índice de riesgo es que puede discriminar entre 
zonas de alto medio y bajo riesgo dentro de las áreas de distribución potencial del género, mientras que otros trabajos (Kapplan et al. 2014; Foxcroft et al. 2006) toman la totalidad del área de distribución potencial como zona de alto riesgo.

Los resultados obtenidos nos permiten decir que el índice desarrollado puede ser extrapolado a cualquier otro taxón, área de estudio y/o escala geográfica si se cuenta con la información de base suficiente para alimentar el modelo. En este sentido, y de acuerdo con lo expuesto por Marchese (2015), un factor limitante en las evaluaciones de conservación es la disponibilidad de datos apropiados y de calidad sobre la información espacial de la que depende la eficacia de la planificación de la conservación. Por tal motivo sería pertinente que los gestores de las unidades de manejo realicen esfuerzos para actualizar las líneas de base de biodiversidad. Es importante destacar que la Argentina cuenta con el Sistema Nacional de Información sobre Especies Exóticas Invasoras, que reúne información sobre especies, localidades de ocurrencia, estado de la invasión e impactos, entre otros datos (www.inbiar.uns.edu.ar), que pueden ser usados como insumos del modelo presentado en este trabajo y para el diseño de estrategias de prevención y control precoz.

Por último, es importante destacar la utilidad de los sistemas de información geográfica para expresar de manera gráfica y sencilla los valores cuantitativos del índice utilizado, lo que permite categorizar la extensión de la invasión de un taxón a nivel regional, nacional o local, en diferentes niveles de riesgo. De todas maneras, y coincidiendo con Kapplan et al. (2014), si bien los mapas de riesgo pueden reducir los costos y el esfuerzo general requeridos para la búsqueda y monitoreo, la participación activa de las partes interesadas en la vigilancia juega un papel decisivo en la detección y delimitación rápida de la extensión de un proceso invasión.

Agradecimientos. Los autores agradecen al CONICET (Consejo Nacional de Investigaciones Científicas y Técnicas, Argentina) y a la Secretaría de Ciencia y Técnica de la Universidad Nacional de Río Cuarto.

\section{REFERENCIAS}

Agencia Córdoba Ambiente D. A. C., and T. S. E. M. 2003. Recursos Naturales de la Provincia de Córdoba. Los Suelos: Nivel de reconocimiento 1:500.000. Dirección de Ambiente, INTA Manfredi. Buenos Aires, Argentina.

Andersen, M. C., H. Adams, B. Hope, and M. Powell. 2004. Risk Analysis for Invasive Species: General Framework and Research Needs. Risk Analysis 24:893-900.

Andreu A. J., and M. Vilà. 2010. Risk analysis of potential invasive plants in Spain. Journal for Nature Conservation 18:34-44.

Australia Weeds Committee. 2007. Weed Identification: Athel Pine. National Weeds Strategy. Natural Resource Management. Ministerial Council, Canberra, Australia.

Bertonatti, C., and J. Corcuera. 2000. Situación Ambiental Argentina 2000. Fundación Vida Silvestre. Buenos Aires, Argentina.

Bilenca, D. N., and F. O. Miñarro (eds.). 2004. Identificación de áreas valiosas de pastizal y campos de Argentina, Uruguay y sur de Brasil. Fundación Vida Silvestre Argentina. Buenos Aires, Argentina.

Brown, A., U. Martínez Ortiz, M. Acerbi, and J. Corcuera (eds.). 2006. La Situación Ambiental Argentina 2005. Fundación Vida Silvestre Argentina. Buenos Aires, Argentina.

Burgman, M. A., D. R. Breininger, B W. Duncan, and S. Ferson. 2001. Setting Reliability Bounds on Habitat Suitability Indices. Ecological Applications 1:70-78.

Burkart, R., N. Bárbaro, R. Sánchez, and D. Gómez. 1996. Eco-Regiones de la Argentina. Administración de Parques Nacionales. Buenos Aires, Argentina.

Chen, C., J. Chen, B. Hu, Y. Jiang, and F. Liu. 2007. Potential Distribution of Alien Invasive Species and Risk Assessment: A Case Study of Erwinia amylovora in China. Agricultural Sciences in China 6:688-605.

Cleverly, J. R., C. N. Dahm, J. R. Thibault, D. J. Gilroy, and J. E. Allred Coonrod. 2002. Seasonal estimates of actual evapo-transpiration from Tamarix ramosissima stands using three-dimensional eddy covariance. Journal of Arid Environments 52:181-197.

Cleverly, J. R., S. D. Smith, S. Sala, and D. A. Devitt. 1997. Invasive capacity of Tamarix ramosissima in a Mojave Desert floodplain: the role of drought. Oecologia 111:12-18.

De Loach, C. J., R. I. Carruthers, J. E. Lovich, T. L. Dudley, and S. D. Smith. 2000. Ecological interactions in the biological control of salt cedar (Tamarix spp.) in the United States: towards a new understanding. Pp. 819-873 en N. R. Spencer (ed.). Proceedings of the X International Symposium on Biological Control of Weeds. Montana State University, Bozeman, Montana.

Di Giacomo, A. (ed.). 2005. Áreas Importantes para la Conservación de las Aves en la Argentina - Sitios prioritarios para la conservación de la biodiversidad. Aves Argentinas. Buenos Aires, Argentina. 
Drolet, D., C. DiBacco, A. Locke, C. H. McKenzie, C. W. McKindsey, A. M. Moore, J. L. Webb, and T. W. Therriault. 2016. Evaluation of a new screening-level risk assessment tool applied to non-indigenous marine invertebrates in Canadian coastal waters. Biological Invasions 18:279-294.

Foxcroft, L. C., M. Rouget, and D. M. Richardson. 2006. Risk Assessment of Riparian Plant Invasions into Protected Areas. Conservation Biology 21:412-421.

Glenn, E. P., and P. M. Nagler. 2005. Comparative ecophysiology of Tamarix ramosissima and native trees in western U.S. riparian zones. Journal of Arid Environments 61:419-446.

Hart, C. H., L. D. White, A. McDonald, and Z. Sheng. 2005. Saltcedar control and water salvage on the Pecos River, Texas, 1999-2003. Journal of Environmental Management 75:399-409.

Horton, J. L., and J. L. C. Forest. 2001. Water table decline alters growth and survival of Salix gooddingii and Tamarix chinensis seedlings. Ecology and Management 140:239-247.

Instituto Nacional de Tecnología Agropecuaria (INTA). 1995. Atlas de Suelos de la República Argentina en CD-Rom con el programa Arc-View incorporado. Instituto de Suelos-CIRN/INTA, AEROTERRA, Fundación ArgenINTA. Buenos Aires, Argentina.

Kaplan, H., A. van Niekerk, J. J. Le Roux, D. M. Richardson, and J. R. U Wilson. 2014. Incorporating risk mapping at multiple spatial scales into eradication management plans. Biological Invasions 16:691-703.

Lawrence, R. W., and Stanley D. S. 1997. Impacts of Invasive Plants on Community and Ecosystem Properties en J. Luken and J. Thieret (eds.). Assessment and Management of Plant Invasion. Springer Series in Environmental Management. EE.UU.

López, C., P. G. Brandolin, O. R. Campanella, A. L. Martino, and C. de Angelo. 2013. Evaluación mediante teledetección del efecto de canalizaciones sobre el humedal del Saladillo, Argentina Revista de Teledetección 40:5-21.

Lovich, J. 1996. A Brief Overview of the Impact of Tamarisk Infestation on Native Plants and Animals. Salt cedar Management Workshop, California, EE.UU.

Mack, R. N., S. Barrett, P. de Fur, W. MacDonald, L. Madden, D. Marshall, D. McCullough, P. McEvay, J. Nyrop, S. Reichard, K. Rice, and S. Tolin. 2002. Predicting Invasions of Nonindigenus Plants and Plant Pest. National Academy Press. Washington, EE. UU.

Malvárez, A. I., and R. F. Bó (compiladores). 2004. Documento del Curso-Taller “Bases ecológicas para la clasificación e inventario de humedales en Argentina". 1a edición. Buenos Aires, Argentina.

Marchese, C. 2015. Biodiversity hotspots: A shortcut for a more complicated concept. Global Ecology and Conservation 3:297-309.

Nagler, P. L., E. P. Glenn, K. Didan, J. Osterberg, F. Jordan, and J. Cunningham. 2008. Wide-Area Estimates of Stand Structure and Water Use of Tamarix spp. on the Lower Colorado River: Implications for Restoration and Water Management Projects. Restoration Ecology 16:136-145.

Natale, E. 2010. Evaluación del riesgo de invasión por Tamariscos (Tamarix spp.) en ambientes naturales y seminaturales de la República Argentina. Tesis para obtener título de Doctor en Ciencias Biológicas. Universidad Nacional de Río Cuarto. Río Cuarto, Córdoba, Argentina.

Natale, E., J. Gaskin, S. M. Zalba, M. Ceballos, and H. Reinoso. 2008. Especies del género Tamarix (Tamarisco) invadiendo ambientes naturales y seminaturales en Argentina. Boletín de la Sociedad Argentina de Botánica 43:137-145.

Natale, E., S. M Zalba, A. Oggero, and H. E. Reinoso. 2010. Establishment of Tamarix ramosissima under different conditions of salinity and water availability: Implications for its management as an invasive species. Journal of Arid Environmental 74:1399-1407.

Natale, E., M. S. Zalba, H. Reinoso, and G. Damilano. 2012. Assessing invasion process through pathway and vector analysis: case of Saltcedar (Tamarix spp.). Management of Biological Invasions 3:37-44.

Natale, E., S. M. Zalba, and H. Reinoso. 2013. Presence-absence versus invasive status data for modelling potential distribution of invasive plants: Saltcedar in Argentina. EcoScience 20:161-171.

Natale, E., and H. Reinoso. 2016. The importance of flood events on the establishment of seedlings and cuttings of saltcedar (Tamarix ramosissima). Ecología Austral 26:001-006.

Neville, D. C., and D. A. Bass. 2008. Application of common predictive habitat techniques for post-border weed risk management. Diversity and Distributions 14:213-224.

Oggero, A., N. De Luca, E. Natale, and M. D. Arana. 2014. Caracterización y situación actual de los bosques nativos en el centro sur de la provincia de Córdoba, Argentina. Voces en el Fénix. ISSN 1853-8819.

Ou, J., C. Lu, and D. K. O'Toole. 2008. A risk assessment system for alien plant bio-invasion in Xiamen, China. Journal of Environmental Sciences 20:989-997.

Owens, M. K., and G. W. Moore. 2007. Saltcedar Water Use: Realistic and Unrealistic Expectations. Rangeland Ecology Manage 60:553-557.

Pertierra, L. R., M. Baker, C. Howard, G. C. Vega, M. A. Olalla-Tarraga, and J. Scott. 2016. Assessing the invasive risk of two non-native Agrostis species on sub-Antarctic Macquarie Island Polar Biology 39:2361-2371.

Porcasi, X., G. E. Calderón, M. Lamfri, M. Scavuzzo, M. S. Sabattini, and J. Polop. 2005. Predictive Distribution Maps of Rodent Reservoir Species of Zoonoses in Southern America. Mastozoología Neotropical 12:199-216.

Singh, S. K., G. J. Ash, and M. Hodda. 2015. Keeping 'one step ahead' of invasive species: using an integrated framework to screen and target species for detailed biosecurity risk assessment. Biological Invasions 17:1069-1086.

Smith, S. D., D. A. Devitt, A. Sala, J. R. Cleverly, and D. E. Busch. 1998. Water relations of riparian plants from warm 
desert regions. Wetlands 18:687-696.

Sobral, R. E., and V. Nakama. 1990. Estado actual de la evaluación de tierras en Argentina. Análisis de los sistemas interpretativos principales. CIRN- INTA-Castelar. Buenos Aires, Argentina.

Stohlgren, T. J., and J. L. Schnase. 2006. Risk Analysis for Biological Hazards: What We Need to Know about Invasive Species Risk Analysis 26:163-173.

Stromberg, J. C., M. K. Chew, P. L. Nagler, and E. P. Glenn. 2009. Changing Perceptions of Change: The Role of Scientists in Tamarix and River Management. Restoration Ecology 17:177-186.

Van Riper III, C., K. L. Paxton, C. O’Brien, P. B. Shafroth, and L. J. McGrath. 2008. Rethinking Avian Response to Tamarix on the Lower Colorado River: A Threshold Hypothesis. Restoration Ecology 16:155-167.

Ward, D. F. 2007. Modelling the potential geographic distribution of invasive ant species in New Zealand. Biological Invasions 9:723-735.

Yemshanov, D., F. H. Koch, D. B. Lyons, M. Ducey, and K. Koehler. 2012. A dominance-based approach to map risks of ecological invasions in the presence of severe uncertainty. Diversity and Distributions 18:33-46.

Zalba, S. M., M. I. Sonaglioni, C. Compagnoni, and C. J. Belenguer. 2000. Assessing the risk of invasion by an exotic plant in a coastal nature reserve. Biological Conservation 93:203-208.

Zavaleta, E. 2000. The Economic Value of Controlling an Invasive Shrub. Ambio 29:462-467. 